\title{
Martin-Dynkin Boundaries of Random Fields
}

\author{
M. Miyamoto \\ Yoshida College, Kyoto University, Kyoto, Japan
}

Received December 20, 1973

\begin{abstract}
Analogous of exit spaces of Dynkin [4] for Markov processes are constructed for random fields introduced by Dobrushin [2].
\end{abstract}

Let $X$ be a finite set and let $T$ be countable. Let $\Omega=X^{T}$ and let $\mathscr{B}_{V}$ be the $\sigma$-algebra generated by $\{\omega \in \Omega ; \omega(t)=x\}_{t \in V, x \in X}$ for $V \subset T$. The $\sigma$-algebra $\mathscr{B}_{T}$ is denoted simply by $\mathscr{B}$. Let be given a system of conditional distributions $q_{V, \omega}(A)$, which satisfy the following conditions, where $V$ is a finite subset of $T, \omega \in \Omega$ and $A \in \mathscr{B}_{V}$.

a) $q_{V, \omega}(\cdot)$ is a probability measure on $\mathscr{B}_{V}$.

B) $q_{V, \omega}(A)$ is a $\mathscr{B}_{V c}$-measurable function of $\omega$ for $A \in \mathscr{B}_{V}$.

$\gamma$ ) If $V_{1} \subset V_{2}$, then for $A \in \mathscr{B}_{V_{1}}, B \in \mathscr{B}_{V_{2} \mid V_{1}}$ and $\omega \in \Omega$

$$
q_{V_{2}, \omega}(A \cap B)=\int_{B} q_{V_{1}, c\left(V_{2} ; \omega^{\prime}, \omega\right)}(A) q_{V_{2}, \omega}\left(d \omega^{\prime}\right),
$$

where $c\left(V_{2} ; \omega^{\prime}, \omega\right)(t)=\omega^{\prime}(t)$ for $t \in V_{2}$, and $=\omega(t)$ for $t \notin V_{2}$.

A probability measure $P$ on $(\Omega, \mathscr{B})$ is called a random field with conditional distribution $q$, if for $A \in \mathscr{B}_{V}$

$$
P\left(A \mid \mathscr{B}_{V c}\right)=q_{V, \omega}(A) \quad \text { a.e. }(P) .
$$

Dobrushin [2] shows that the totality $\mathscr{P}$ of random fields with $q$ is a non-empty, compact and convex set, if

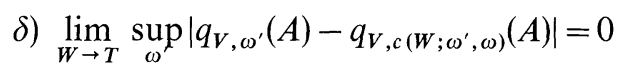

for $A \in \mathscr{B}_{V}$ and $\omega \in \Omega$, which we assume throughout this note.

Let $V_{1} \subset V_{2} \subset \cdots$ be an increasing sequence of finite subsets $V_{n}$ of $T$ with $\cup V_{n}=T$. Let $\Omega_{\infty}$ be the set of $\omega$ for which there exists $\lim _{n \rightarrow \infty} q_{V_{n}, \omega}(A)$ for every cylindrical $A$.

Let $Q_{\omega}(A)$ be the limit. $Q_{\omega}(\cdot)$ is countably additive on $\mathscr{B}_{V}$ for every finite subset $V$. Therefore it is extended to a probability measure on $\mathscr{B}$, which we denote by the same $Q_{\omega}$. It is easy to see $Q_{\omega} \in \mathscr{P}$.

Let $\mathscr{B}_{\infty}=\bigcap_{V} \mathscr{B}_{V c}$, where $V$ runs over the set of all finite subsets of $T$. 
Lemma 1. If $P \in \mathscr{P}$, then i.e.,

$$
P(A \cap B)=\int_{B} Q_{\omega}(A) P(d \omega) \quad \text { for } \quad A \in \mathscr{B} \quad \text { and } B \in \mathscr{B}_{\infty},
$$

$$
P\left(A \mid \mathscr{B}_{\infty}\right)=Q_{\omega}(A) \text { a.e. }(P) .
$$

Proof. Taking in mind that $q_{V_{n}, \omega}(A)=P\left(A \mid \mathscr{B}_{V_{n}^{c}}\right)$ is a martingale, we have $P\left(\Omega_{\infty}\right)=1$ for every random field $P$ by Doob's convergence theorem [3]. If $A \in \mathscr{B}_{V_{n}}$ and $B \in \mathscr{B}_{\infty}$, then $P(A \cap B)=\int_{B} q_{V_{n}, \omega}(A) P(d \omega)$ $=\int_{B \cap \Omega_{\infty}} q_{V_{n}, \omega}(A) P(d \omega)$. Letting $n \rightarrow \infty$, we have

$$
P(A \cap B)=\int_{B \cap \Omega_{\infty}} Q_{\omega}(A) P(d \omega)=\int_{B} Q_{\omega}(A) P(d \omega) .
$$

The equality holds also for non-cylindrical $A$.

Let $2_{P}=\{A ; P(A)=0$ or 1$\}$.

Lemma 2. (Theorem 3.4 in [6].) $P$ is extremal in $\mathscr{P}$ if and only if $\mathscr{B}_{\infty}=2_{p}(\bmod P)$.

Proof. i) We assume $\mathscr{B}_{\infty} \neq 2_{p}(\bmod P)$, then there exists $\tilde{\Omega} \in \mathscr{B}_{\infty}$ such that $0<P(\tilde{\Omega})<1$. Let $P_{\tilde{\Omega}}(\cdot)=P(\tilde{\Omega})^{-1} P(\cdot \cap \tilde{\Omega})$. For every $A \in \mathscr{B}_{V}$ we have $P_{\widetilde{\Omega}}\left(A \mid \mathscr{B}_{V^{c}}\right)=q_{V, \omega}(A)$, i.e., $P_{\widetilde{\Omega}} \in \mathscr{P}$, because for every $B \in \mathscr{B}_{V^{c}}$,

$$
\begin{aligned}
P_{\widetilde{\Omega}}(A \cap B) & =P(\tilde{\Omega})^{-1} P(A \cap B \cap \tilde{\Omega})=P(\tilde{\Omega})^{-1} \int_{B \cap \tilde{\Omega}} P\left(A \mid \mathscr{B}_{V^{c}}\right) P(d \omega) \\
& =\int_{B} q_{V, \omega}(A) P(\tilde{\Omega})^{-1} P(d \omega \cap \tilde{\Omega})=\int_{B} q_{V, \omega}(A) P_{\widetilde{\Omega}}(d \omega) .
\end{aligned}
$$

A measure $P_{\widetilde{\Omega}^{c}} \in \mathscr{P}$ is defined analogously. Both $P_{\widetilde{\Omega}}$ and $P_{\widetilde{\Omega}^{c}}$ are distinct, since they are mutually singular. Therefore the sum $P=P(\tilde{\Omega}) P_{\tilde{\Omega}}$ $+P\left(\tilde{\Omega}^{c}\right) P_{\Omega^{c}}$ is not extremal.

ii) Let $\mathscr{B}_{\infty}=2_{P}(\bmod P)$ and let $P=\lambda P_{1}+(1-\lambda) P_{2}$, where $0<\lambda<1$ and $P_{1}, P_{2} \in \mathscr{P}$. By Lemma $1, Q_{\omega}(A)=P(A)$ a.e. $(P)$ for each $A \in \mathscr{B}$, that is, $P\left\{\omega: P(A)=Q_{\omega}(A)\right\}=1$, hence $P_{i}\left\{\omega: Q_{\omega}(A)=P(A)\right\}=1$ for $i=1,2$, because the coefficients $\lambda$ and $1-\lambda$ are both positive. Thus we have, by Lemma 1 again, $P_{i}(A)=\int Q_{\omega}(A) P_{i}(d \omega)=P(A)$, that is, $P_{i}=P$, therefore $P$ is extremal.

Corollary. $P$ is extremal if and only if

$$
\lim _{V \rightarrow T} \sup _{B \in \mathscr{B}_{V c}}|P(A \cap B)-P(A) P(B)|=0 \text { for all } A \in \mathscr{B} .
$$

Let $B_{\omega}=\left\{\omega^{\prime} ; Q_{\omega^{\prime}}=Q_{\omega}\right\}$, which belongs to $\mathscr{B}_{\infty}$, as is easily seen. We have, by Lemma 1, $Q_{\omega}\left(B_{\omega}\right)=Q_{\omega}\left(B_{\omega} \cap B_{\omega}\right)=\int_{B_{\omega}} Q_{\omega^{\prime}}\left(B_{\omega}\right) Q_{\omega}\left(d \omega^{\prime}\right)$ $=Q_{\omega}\left(B_{\omega}\right)^{2}$, so that $Q_{\omega}\left(B_{\omega}\right)=0$ or 1 . We call $\omega$ regular, if $Q_{\omega}\left(B_{\omega}\right)=1$. Let $\Omega_{r}$ be the set of all regular $\omega$. 
Theorem 1. $P$ is extremal in $\mathscr{P}$ if and only if $P=Q_{\omega}$ for some $\omega \in \Omega_{r}$. (Cf. Theorem 2.2 in [5].)

Proof. i) We assume that $P$ is extremal, i.e., $\mathscr{B}_{\infty}=2_{\boldsymbol{P}}(\bmod P)$ by Lemma 2. Since $Q_{\omega^{\prime}}(A)$ is $\mathscr{B}_{\infty}$-measurable, $Q_{\omega^{\prime}}(A)$ does not depend on $\omega^{\prime}$ a.e. $(P)$, so that there exists $\Omega_{A}$ with $P\left(\Omega_{A}\right)=1$ for whose elements $\omega_{1}$ and $\omega_{2}$ it holds $Q_{\omega_{1}}(A)=Q_{\omega_{2}}(A)$. Let $\bar{\Omega}=\bigcap_{A} \Omega_{A}$, where $A$ runs over the set of all cylindrical subsets of $\Omega$. The number of cylindrical subsets of $\Omega$ is countable, hence $P(\bar{\Omega})=1$. Take an arbitrary element $\omega$ of $\bar{\Omega}$, then $Q_{\omega^{\prime}}=Q_{\omega}$ for almost all $(P) \omega^{\prime}$. By Lemma 1 we have $P=\int Q_{\omega^{\prime}} P\left(d \omega^{\prime}\right)=Q_{\omega^{\prime}}$.

If $\omega$ is not regular, then $P\left(B_{\omega}\right)=Q_{\omega}\left(B_{\omega}\right)=0$, i.e., $P\left(B_{\omega}^{c}\right)=P\left\{\omega^{\prime} ; Q_{\omega^{\prime}} \neq Q_{\omega}\right\}$ $=P\left\{\omega^{\prime} ; Q_{\omega^{\prime}} \neq P\right\}=1$. Therefore we have $P\left\{\omega^{\prime}: Q_{\omega^{\prime}}(A) \neq P(A)\right\}>0$ for some cylindrical $A$, which implies $P\left\{\omega^{\prime}: Q_{\omega^{\prime}}(A)>P(A)\right\}>0$ or $P\left\{\omega^{\prime}: Q_{\omega^{\prime}}(A)<P(A)\right\}>0$.

In case when $P\left\{Q_{\omega^{\prime}}(A)>P(A)\right\}>0$, we have $P\left\{Q_{\omega^{\prime}}(A)>P(A)\right\}=1$ by our assumption that $\mathscr{B}_{\infty}=2_{P}(\bmod P)$. By Lemma $1, P(A)=\int Q_{\omega^{\prime}}(A)$ $\cdot P\left(d \omega^{\prime}\right)=\int_{\left\{\omega^{\prime}(A)>P(A)\right\}} Q_{\omega^{\prime}}(A) P\left(d \omega^{\prime}\right)>P(A)$, which is absurd. In case when $P\left\{Q_{\omega^{\prime}}(A)<P(A)\right\}>0$, we are led to the same contradiction.

ii) Let $P=Q_{\omega}$ for some $\omega \in \Omega_{r}$. For $A \in \mathscr{B}_{\infty}$ we have

$$
\begin{aligned}
P(A)=Q_{\omega}\left(A \cap A \cap B_{\omega}\right) & =\int_{A \cap B_{\omega}} Q_{\omega^{\prime}}(A) Q_{\omega}\left(d \omega^{\prime}\right)=\int_{A} Q_{\omega}(A) Q_{\omega}\left(d \omega^{\prime}\right) \\
& =Q_{\omega}(A)^{2}=P(A)^{2} .
\end{aligned}
$$

Therefore $P(A)=0$ or 1 for $A \in \mathscr{B}_{\infty}$, i.e., $\mathscr{B}_{\infty}=2_{P}(\bmod P)$.

Corollary 1. $P\left(\Omega_{r}\right)=1$ for all $P \in \mathscr{P}$.

Proof. The Choquet theorem [1] shows that any $P \in \mathscr{P}$ is represented in the form; $P=\int_{\Omega_{r}} Q_{\omega} \mu(d \omega)$. For any regular $\omega, Q_{\omega}\left(\Omega_{r}\right)=1$, since $B_{\omega} \subset \Omega_{r}$. Therefore $P\left(\Omega_{r}\right)=\int_{\Omega_{r}} Q_{\omega}\left(\Omega_{r}\right) \mu(d \omega)=1$.

Corollary 2. Extremal random fields of $\mathscr{P}$ are mutually singular.

Let $\mathscr{B}_{r}$ be the $\sigma$-algebra on $\Omega_{r}$ generated by $\left\{Q_{\omega}(A) ; A \in \mathscr{B}\right\}$.

Lemma 3. The $\sigma$-algebra $\mathscr{B}_{r}$ coincides with the family of sets in $\mathscr{B}_{\infty}$ which are representable as a (possibly uncountable) union of sets $B_{\omega}$ for regular $\omega$.

Proof. If $\cup B_{\omega}$ belongs to $\mathscr{B}_{\infty}$, then $Q_{\omega^{\prime}}\left(\cup B_{\omega}\right)=\chi_{\cup B_{\omega}}\left(\omega^{\prime}\right)$. Therefore $\cup B_{\omega} \in \mathscr{B}_{r}$. On the other hand $\left\{\omega: Q_{\omega}(A)<a\right\}=\bigcup_{\omega ; Q_{\omega}(A)<a} B_{\omega} \in \mathscr{B}_{\infty}$, from which follows our result.

Let $\left.P\right|_{r}$ be the restriction of $P$ on $\mathscr{B}_{r}$. 
Theorem 2. For any $P \in \mathscr{P}, P=\left.\int_{\Omega_{r}} Q_{\omega} P\right|_{r}(d \omega)$. If $P=\int_{\Omega_{r}} Q_{\omega} \mu(d \omega)$ with a probability measure $\mu$ on $\left(\Omega_{r}, \mathscr{B}_{r}\right)$, then $P \in \mathscr{P}$ and $\mu=\left.P\right|_{r}$. (Cf. Proposition 3.5 in [6].)

Proof. $P=\left.\int_{\Omega_{r}} Q_{\omega} P\right|_{r}(d \omega)$ is a direct consequence of Lemma 1 and Corollary 1 to Theorem 1 . Let $P=\int_{\Omega_{r}} Q_{\omega^{\prime}} \mu\left(d \omega^{\prime}\right)$ and let $\cup B_{\omega} \in \mathscr{B}_{r}$. We have $P\left(\cup B_{\omega}\right)=\int_{\Omega_{r}} Q_{\omega^{\prime}}\left(\cup B_{\omega}\right) \mu\left(d \omega^{\prime}\right)=\int_{\Omega_{r}} \chi_{\cup B_{\omega}}\left(\omega^{\prime}\right) \mu\left(d \omega^{\prime}\right)=\mu\left(\cup B_{\omega}\right)$, hence $P=\mu$ on $\mathscr{B}_{r}$ by Lemma 3 .

Let us consider a case where $T$ is the $v$-dimensional lattice $Z^{v}$. For $\tau \in T$, let notations be as follows:

$$
\begin{array}{rll}
\tau V=\{\tau+v ; v \in V\} & \text { for } & V \subset T, \\
\tau \omega(t)=\omega(t-\tau) & \text { for } & \omega \in \Omega \text { and } t \in T, \\
\tau A=\{\tau \omega ; \omega \in A\} & \text { for } & A \in \mathscr{B} .
\end{array}
$$

Let $S$ be a subgroup of $T$ and let conditional distributions $q_{V, \omega}$ be $S$ invariant, i.e., $q_{\tau V, \tau \omega}(\tau A)=q_{V, \omega}(A)$ for all $\tau \in S$. We slightly modify the definition of $\Omega_{\infty}$ and $Q_{\omega}$; let $\Omega_{\infty}$ be the set of $\omega$ for which there exists $\lim _{n \rightarrow \infty} q_{\tau V_{n}, \omega}(A)$ for every cylindrical $A$ and every $\tau \in S$ and these limits coincide with each other for all $\tau \in S$. The limit is denoted by $Q_{\omega}$ for $\omega \in \Omega_{\infty}$. The same convergence theorem as in the proof of Lemma 1 assures that $P\left(\Omega_{\infty}\right)=1$ for each $P \in \mathscr{P}$. Corresponding modifications are made for definitions of $B_{\omega}, \Omega_{r}$, etc. The same argument as preceding one works for our modified $\Omega_{\infty}, \Omega_{r}, B_{\omega}$, etc. Obviously, $Q_{\omega}(\tau A)$ $=\lim _{n \rightarrow \infty} q_{V_{n}, \omega}(\tau A)=\lim _{n \rightarrow \infty} q_{\tau^{-1} V_{n}, \tau^{-1} \omega}(A)=Q_{\tau^{-1} \omega}(A)$ for $\omega \in \Omega_{\infty}$ and $\tau \in S$. It is easy to see that $\Omega_{r}$ is $S$-invariant. Finally we remark that $P=\int_{\Omega_{r}} Q_{\omega} \mu(d \omega)$ is $S$-invariant if and only if $\mu$ is so.

\section{References}

1. Choquet, G.: Ann. Inst. Fourier 10, 333-344 (1960)

2. Dobruschin, R. L.: Teor. Verojatnost. i Primenen 13, $201-229$ (1968) Russian

3. Doob, J. L.: Stochastic Processes 1953

4. Dynkin,E. B.: Actes congrès internat. math., tom 2, 507-512 (1970)

5. Georgii, H. O.: Commun. math. Phys. 32, 107-118 (1973)

6. Lanford III, O.E., Ruelle, D.: Commun. math. Phys. 13, 194-215 (1969)

Communicated by G. Gallavotti

M. Miyamoto

Yoshida College

Kyoto University

Kyoto, Japan 